\title{
The first report of co-existence of pulmonary tuberculosis and lung malignancy in a kidney transplant recipient: a case report and literature review
}

\author{
Gang Fang ${ }^{1 \dagger}$, Ning-Chang Cheng ${ }^{2 \dagger}$, Li-Li Huang ${ }^{1}$, Wei-Ping Xie ${ }^{3}$, Chun-Mei Hu ${ }^{1 *}$ and Wei Chen ${ }^{4 *}$ (1)
}

\begin{abstract}
Background: Along with the medical development, organ transplant patients increase dramatically. Since these transplant patients take immunosuppressants for a long term, their immune functions are in a suppressed state, prone to all kinds of opportunistic infections and cancer. However, it is rarely reported that the kidney transplant recipients (KTRs) have pulmonary tuberculosis and lung cancer simultaneously.

Case presentation: A 60-year-old male was admitted because of persistent lung shadow for 2 years without any obvious symptom 8 years after renal transplant. T-SPOT test was positive but other etiological examinations for Mycobacterium tuberculosis were negative. Chest $\mathrm{CT}$ scan revealed two pulmonary lesions in the right upper and lower lobe respectively. ${ }^{18}$ F-fluorodesoxyglucose positron-emission tomography (FDG-PET) CT found FDG intake increased in both pulmonary consolidation lesions. CT-guided percutaneous transthoracic needle biopsy revealed lung adenocarcinoma and tuberculosis. The video-assisted thoracoscopic surgery was operated to resect the malignancy lesions. The patient received specific anti-tuberculosis therapy and was discharged. At the follow-up of 6 months post drug withdrawal, the patient was recovered very well.

Conclusions: We for the first time reported co-existence of smear-negative pulmonary TB and lung adenocarcinoma in a KTR, which highlighted the clinical awareness of co-occurrence of TB and malignancy after renal transplant and emphasized the value of biopsy and ${ }^{18} \mathrm{~F}-\mathrm{FDG}-\mathrm{PET}$ in early diagnosis of TB and cancer.
\end{abstract}

Keywords: Kidney transplant recipient, Tuberculosis, Lung cancer, Co-existence

\section{Background}

Kidney transplant is an important surgical procedure to treat kidney failure, improving and prolonging the life of patients. However, successful organ transplant faces a lot of challenges, since many postoperative complications

\footnotetext{
* Correspondence: hcm200702@163.com; weichennannan2017@163.com

${ }^{\dagger}$ Gang Fang and Ning-Chang Cheng contributed equally to this work.

'Department of Tuberculosis, The Second Hospital of Nanjing, Nanjing University of Chinese Medicine, Nanjing 210003, China

${ }^{4}$ Clinical Research Center, The Second Hospital of Nanjing, Nanjing University of Chinese Medicine, Nanjing 210003, China

Full list of author information is available at the end of the article
}

might occur $[1,2]$. Among all the infective complications, tuberculosis (TB) is the most common one after renal transplantation. The pooled prevalence of $\mathrm{TB}$ included active TB and latent TB infection in kidney transplant recipients (KTRs) is $2.51 \%$ worldwide, and is even higher in the developing countries [3, 4]. Post-transplant $\mathrm{TB}$ is considered as a major problem in successful longterm outcome of KTRs [5]. Besides TB, cancer is another important post-transplant complication, which is the second cause of mortality and morbidity in KTRs [6]. In 2019, a single center analysis showed that 39 of

(c) The Author(s). 2021 Open Access This article is licensed under a Creative Commons Attribution 4.0 International License, which permits use, sharing, adaptation, distribution and reproduction in any medium or format, as long as you give appropriate credit to the original author(s) and the source, provide a link to the Creative Commons licence, and indicate if changes were made. The images or other third party material in this article are included in the article's Creative Commons licence, unless indicated otherwise in a credit line to the material. If material is not included in the article's Creative Commons licence and your intended use is not permitted by statutory regulation or exceeds the permitted use, you will need to obtain permission directly from the copyright holder. To view a copy of this licence, visit http://creativecommons.org/licenses/by/4.0/. The Creative Commons Public Domain Dedication waiver (http://creativecommons.org/publicdomain/zero/1.0/) applies to the data made available in this article, unless otherwise stated in a credit line to the data. 
535 (7.2\%) KTRs developed malignancies within a mean follow-up of 7.8 years [7]. Unfortunately, the prognosis of KTRs with malignancies is not good. Almost half of them die with the average age 58.5 [7].

As we know, KTRs are under immunosuppressed conditions [8]. Even though pre-transplant screening of both donor and recipient for $\mathrm{TB}$ and cancer is conducted, reactivation of latent TB, new TB infection or de novo malignancy appear inevitably in some cases. Prompt diagnosis and treatment is pivotal to extend the healthy and productive life of KTRs. However, among different $\mathrm{TB}$ types, smear- or culture-negative $\mathrm{TB}$ is especially hard to make a definite diagnosis. Additionally, the clinical and radiological features between TB and cancer are very similar, which often leads to misdiagnosis and delayed treatment [9]. Thus, every case with definite diagnosis and different characteristics is valuable for the clinicians. So far, even abundant cases about complicated TB or malignancy among KTRs are already reported, there is no combination of pulmonary TB and lung malignancy in KTRs, which dramatically challenges the success of allograft.

In this case, we for the first time reported co-existence of smear-negative pulmonary TB and lung adenocarcinoma in a KTR, which highlighted the clinical awareness of co-occurrence of TB and malignancy after renal transplant and emphasized the value of biopsy and ${ }^{18} \mathrm{~F}$-FDGPET in early diagnosis of TB and cancer.

\section{Case presentation}

In December 2019, a 60-year-old man was admitted to the Second Hospital of Nanjing due to persistent lung shadow for 2 years, but without any clinical symptoms. He had received the allograft kidney transplant because of chronic renal failure since 2011. The regular pretransplant screening reports ever excluded TB or cancer for both the donor and the receipt. Afterwards, the renal function of the patient maintained stable with the combination drug therapy, which was composed of mycophenolate mofetil ( $500 \mathrm{mg} / 250 \mathrm{mg}$ per day), cyclosporine (75 $\mathrm{mg}$ twice per day), prednisone ( $5 \mathrm{mg}$ once per day), phoebus, Bailing capsule and valsartan. In December 2017, during the follow-up examination in the kidney transplant hospital, the chest CT found speckle and stripe shadows in the posterior segment of the right upper lobe (Fig. 1A), and a nodule in the anterior segment of right lower lobe with small cavity shadow inside (Fig. 1B). Both T-SPOT and purified protein derivative (PPD) tests were positive for Mycobacterium tuberculosis (MTB) infection, but detection of MTB from the sputum samples through smear microscopy, culture and realtime PCR was negative. Then, the empirical anti-TB therapy was started. Whereas, this treatment was terminated after 2 weeks since the patient family worried about indefinite diagnosis of TB and side effects.

At admission, physical examination revealed that respiratory, cardiovascular, abdominal and neurological characteristics were normal. Complete blood count test, C-reaction protein, erythrocyte sedimentation rate, coagulation (D-Dimer), tumor markers, liver and renal function tests were negative. As before, T-SPOT test was positive, but smear microscopy, culture and realtime PCR for MTB were still negative.

Remarkably, chest CT found some changes compared with 2 years ago. In the posterior segment of the right upper lobe, a soft tissue density mass shadow with spiculate boundary appeared (Fig. 1C). Also, the nodule in the right lower lobe became bigger (Fig. 1D). According to those radiological findings, the possibility of lung cancer in the upper lobe was considered. ${ }^{18} \mathrm{~F}$-fluorodesoxyglucose positron-emission tomography (FDG-PET) CT found that FDG intake increased in both the pulmonary consolidation lesions, with SUVmax 8.2 in the right upper lobe and SUVmax 1.9 in the right lower lobe, respectively (Fig. 2). Meanwhile, there was no obvious abnormality for the remaining part of lung, mediastinal lymph nodes, liver and kidney. Taken together, adenocarcinoma was probably considered. A CT-guided percutaneous transthoracic needle biopsy was then operated, and the pathological result confirmed lung adenocarcinoma in the right upper lobe.

The video-assisted thoracoscopic surgery (VATS) right upper lobectomy, wedge resection of right lower lobe and lymphadenectomy were operated. The pathological results revealed that both lobes had invasive adenocarcinoma but with different histological subtypes, according to the predominant pattern of the neoplastic cell growth, which was mainly acinar type and solid type for the upper lobe and lower lobe, respectively (Fig. 3A and B). To our interest, we observed caseous necrosis along with acinar type adenocarcinoma in the right upper lobe (Fig. 3A). Acid-fast staining for the surgical tissue samples detected MTB in the field (Fig. 4). Real-time PCR was also positive for MTB. Therefore, we could make a definite diagnosis that smear-negative TB and adenocarcinoma co-existed in the right upper lobe.

According to TNM classification criteria, the adenocarcinoma in the right lobe was diagnosed with Stage IA, no chemotherapy or radiotherapy was taken further after surgical resection. Since TB infection was complicated in the right upper lobe, anti-TB treatment was still necessary. However, the challenge for TB treatment was how to avoid potential renal toxicity of anti-TB drugs. Considered that the KTR has been taking anti-rejection medication, rifampicin was firstly excluded because it would affect the expression of cytochrome P450 3A4 in the liver and in the small intestine and interfere with the 
A

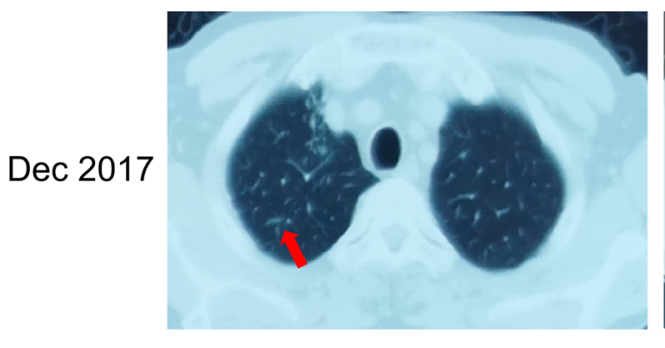

C

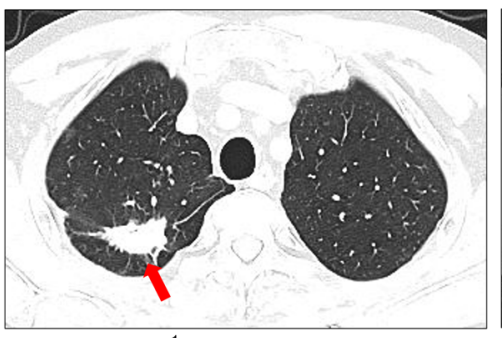

E

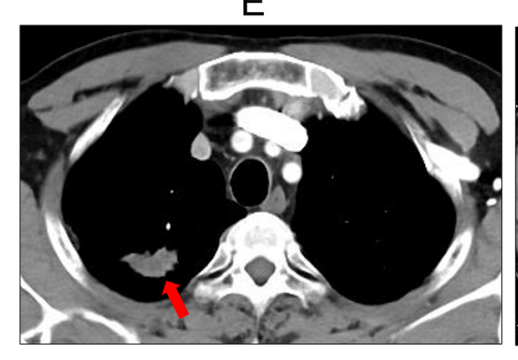

G

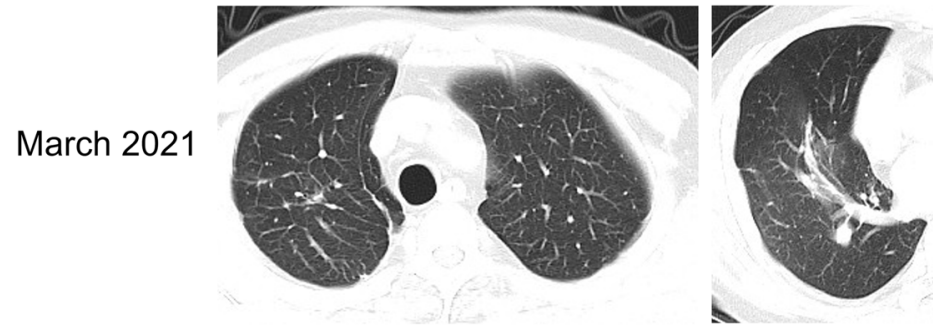

B

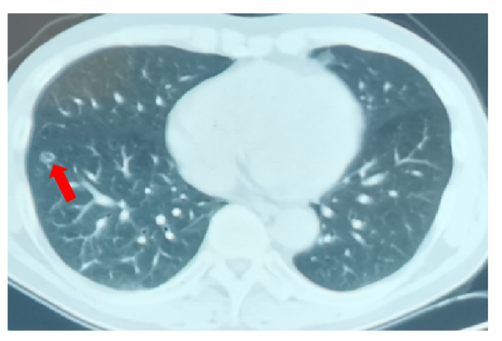

D

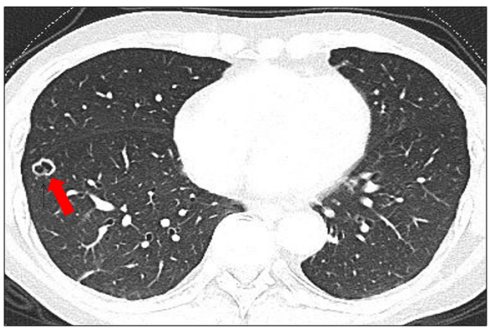

F

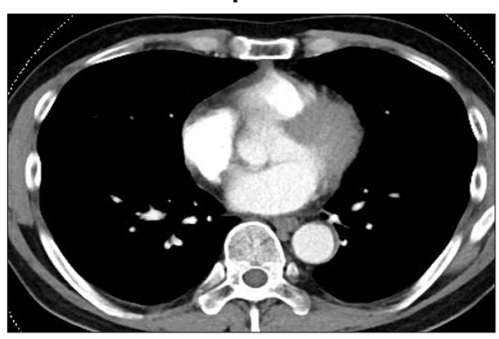

$\mathrm{H}$

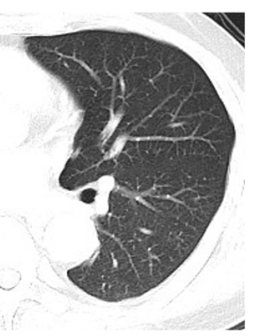

Fig. 1 Chest CT at different time points. In December 2017, chest CT revealed speckle and stripe shadows in the posterior segment of the right upper lobe $(\mathbf{A})$ and a nodule in the anterior segment of right lower lobe with small cavity shadow inside (B). At admission, chest CT found a soft tissue density mass shadow $(30 \mathrm{~mm} \times 19.6 \mathrm{~mm}$ ) with spiculate boundary in the posterior segment of the right upper lobe (C) and the nodule (11 $\mathrm{mm} \times 11 \mathrm{~mm}$ ) in the right lower lobe became bigger than two years ago (D). The mass shadow was observed in the mediastinal window (E) but not for the nodule (F). At the follow-up of 6 months post drug withdrawal, after resection of the original mass, a few cord shadows were seen in the posterior apical segment of the right upper lobe $(\mathbf{H})$. After wedge-shaped surgery of the right lower lobe to excise of the original cavitation nodule in the anterior basal segment of the right lower lobe, a few stripe shadows were observed (G). Lesions were indicated by red arrows

metabolism of anti-rejection drugs [10]. Similarly, pyrazinamide was also excluded as it would lead to hyperuricemia and influence the renal function. The final anti$\mathrm{TB}$ regimen for this patient was isoniazid $(0.3 \mathrm{mg}$, QD, ethambutol (0.75 mg, QD) and moxifloxacin (0.4 mg, QD). After two-week treatment in hospital, the patient was discharged for continuous anti-TB treatment for 9 months in total.

In March 2021, at 6-month follow-up after drug withdrawal, the patient kept healthy without any uncomfortable symptoms. The normal tests for MTB and tumor were all negative, and the renal function was normal. 

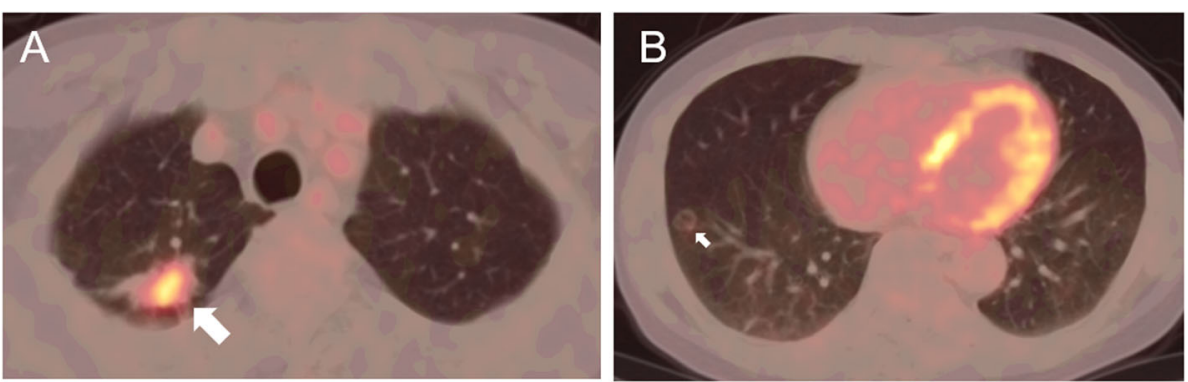

Fig. 2 FDG-PET CT. FDG intake increased in both the pulmonary consolidation lesions, with SUVmax 8.2 in the right upper lobe and SUVmax 1.9 in the right lower lobe, respectively. Lesions with increased FDG intake were indicated by arrows

\section{Discussion and conclusion}

\section{Characteristics of KTRs complicated with TB or cancer}

We performed literature searching in PubMed database with the keywords: (tuberculosis) AND (lung cancer OR lung carcinoma) AND (kidney transplant OR renal transplant). The search was limited to English literature and other language literature with English abstract. To our best knowledge, this was the first time to report coexistence of pulmonary tuberculosis and lung malignancy in a kidney transplant recipient. However, when using Mesh terms "kidney transplant" \& "tuberculosis" (KT/TB) from 2016 to 2020, and finally found 21 cases (Table S1). The KT/TB patients were mainly middleaged, with the median age 48.29. Gender was not associated with $\mathrm{TB}$ incidence. Of $21 \mathrm{KT} / \mathrm{TB}$ patients, 10 (47.6\%) had none underlying disease. Among the remaining 11 patients, 5 had diabetes and 3 had hypertension. Meanwhile, extra-pulmonary TB was predominant, with 17 of 21 cases (80.95\%). The median onset time of TB was 7 months post transplant, with the shortest 3 months and the longest 12 years. Diagnosis of TB mostly depended on culture of MTB and qPCR for MTB DNA through the biopsy samples from lesions. RIPE scheme (rifampicin, isoniazid, pyrazinamide and ethambutol) was the most common anti-TB therapy (10/21, $47.62 \%)$. The prognosis of KT/TB patients seemed to be optimistic.

As well, the literatures about KT complicated with lung cancer (LC) from 2016 to 2020 were summarized in Table S2. In China, a single-center analysis reported that $0.5 \%$ of KTR developed lung cancer from 1988 to 2015 in a southern hospital of China [11]. The mean age of KT/LC patients was $50.2 \pm 8.3$ years at transplantation. The average interval from renal transplantation to cancer diagnosis was $65.7 \pm 20.1$ months. In France, a casecontrol study analyzed retrospectively all $\mathrm{KT} / \mathrm{LC}$ cases in three French Renal Transplant Units from 2003 to 2012 and found that LC incidence was 1.89/1000 person-years
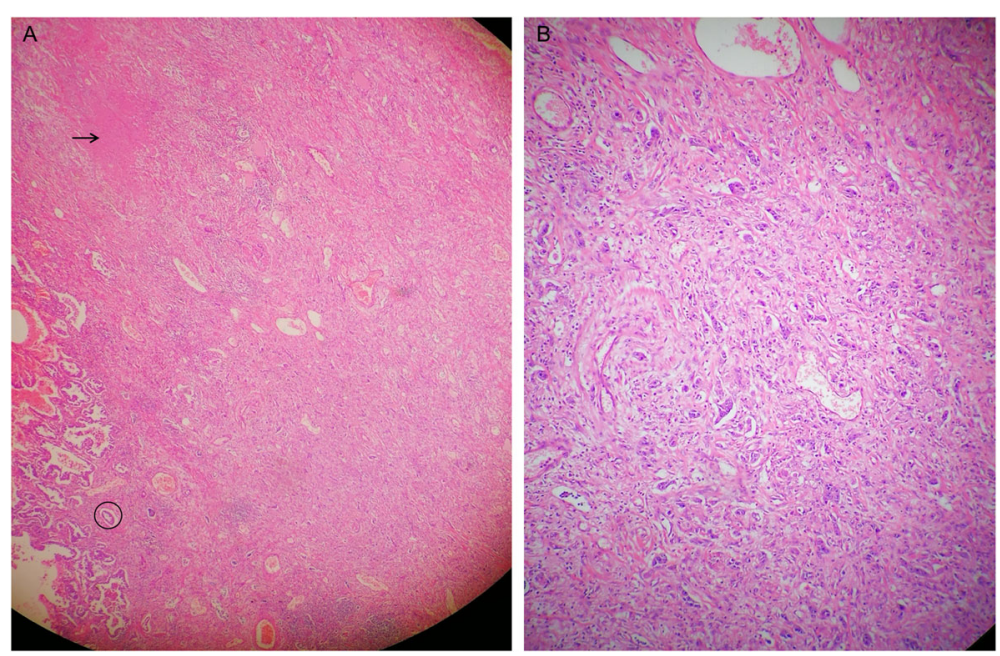

Fig. 3 Pathological examination of tissue specimens from two consolidation lesions. A The right upper lobe had invasive adenocarcinoma, grade II-III, mainly acinar type (90\%), with the size of $2.5 \mathrm{~cm} \times 1.8 \mathrm{~cm} \times 1.5 \mathrm{~cm}$ (indicated by black circle). The tumor was peripheral type, which did not break through the pulmonary capsule. Sprinkle multifocal epithelioid granulomatous nodules were seen in the surrounding lung tissues (indicated by black arrow). Caseous necrosis formed along with multinucleated giant cells. B The right lower lobe had infiltrating adenocarcinoma, grade III, mainly solid type, with the size of $1.2 \mathrm{~cm} \times 0.9 \mathrm{~cm} \times 0.7 \mathrm{~cm}$. No cancer tissue was found in the lung membrane 


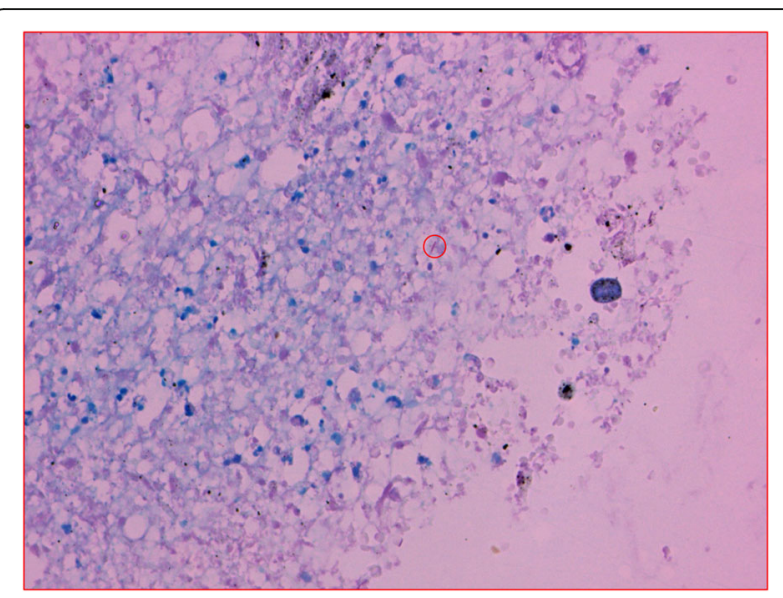

Fig. 4 Acid-fast staining to detect MTB for the tissue specimen of the right upper lobe. Only one MTB was observed in the sight field, which was indicated by a red circle

from 2008 to 2012 [12]. The median interval of time from KT to LC diagnosis was 7 years (range 0.5-47 years). In our case, the adenocarcinoma in the right lung might occur 6 years post KT.

\section{Relationship between TB and cancer}

As we know, TB is the major public health threat of human, causing approximately 10 million new infection and 1.2 million death among HIV-negative people worldwide in 2019 [13]. In the meantime, based on the data of "Statista" website, lung cancer has the highest rate of death among different cancer types and causes 23 death per 100,000 population in the world in 2020 . Actually, co-existence of TB and lung cancer has been well-described [14, 15]. At one hand, inflammatory process is one of the potential factors of cancer. Pulmonary $\mathrm{TB}$ is considered as the most important inflammation-inducing factors in the lungs $[16,17]$. Additionally, it's proposed that TB could cause chromosome breakage of bronchial epithelium and the bacterial DNA could also integrate into the chromosome of cells, leading to neoplastic transformation [15]. At the other hand, lung cancer may cause re-activation or recurrence of latent TB in turn $[18,19]$. Immunotherapy of cancer often includes immunosuppressors, predisposing the patients to an immunocompromised state that latent tuberculosis infection is reactivated [20].

Although both the right upper and lower lung were adenocarcinoma, but the specific subtype was different. After kidney transplantation, the patient took immunosuppressant and anti-rejection drugs for a long term. Thus, he was at a status of low immunity and weak autoimmune monitoring, who was prone to develop cancer. In this case, TB was diagnosed earlier and the cancer was at stage IA. We inferred that $\mathrm{TB}$ developed first and induced neoplastic transformation. The cancer cells may grow adherently along the acinar duct, resulting in obvious enlargement of the lesion 2 years later. However, the right lower cavity-like nodules develop more slowly in comparison. Because the causes are not exactly the same, the subtypes are different, and ultimately the prognosis and course of the disease are not necessarily the same.

\section{Challenges on definite diagnosis of TB and lung cancer}

Because most individuals presented with atypical and unspecific symptoms and the radio-logical findings between TB and lung cancer were very similar, challenges remain on diagnosing co-existence of tuberculosis and lung cancer $[14,21]$. In this case, the presence of cavitary lesion on the chest CT image indicated possible TB infection. Nevertheless, cavities on thoracic images occasionally indicated various infections, autoimmune conditions, or primary and metastatic malignancies [21]. Moreover, the heterogeneous pattern of FDG uptake increase may provide clues to the presence of double pathologies: infection and malignancy [22]. Therefore, CT-guided PTNB was applied for definite diagnosis, which provided high accuracy, sensitivity, and specificity for both malignancy and infection [23]. A recent research revealed that CT-guided PTNB had overall diagnostic accuracy of $83 \%(19 / 20)$ in the evaluation of pulmonary consolidation lesions [24].

\section{Challenges on anti-TB treatment in KTRs}

Anti-TB medications in KTRs caused a high mortality from 12 to $16.7 \%$ all over the world [25]. In addition, drug-drug interactions with immunosuppressive drugs might lead to potential renal or liver toxicity [26]. The well-known "first line" anti-TB regimen includes isoniazid, rifampicin, pyrazinamide and ethambutol. In this case, rifampicin was firstly excluded because of its renal toxicity. Pyrazinamide was not used as it would lead to hyperuricemia and influence the renal function. A recent retrospective study proved that concurrent chemotherapy (anticancer and anti-TB chemotherapy) was effective and safe for treating cancer patients with active TB [27]. However, immune checkpoint inhibitors such as PD1/PD-L1 were widely used on cancer treatment, which may induce reactivation of LTBI or develop active TB [28]. Fortunately, this patient only required anti-TB therapy since the cancer lesions were resected by surgery.

In conclusion, this case described a rare phenomenon of co-existence of smear-negative pulmonary TB and lung adenocarcinoma in KTRs, which highlighted the 
clinical awareness of co-occurrence of TB and de novo malignancy after renal transplant and emphasized the value of biopsy and ${ }^{18}$ F-FDG-PET in early diagnosis of $\mathrm{TB}$ and cancer. The anti-TB therapy should consider the complicated drug-drug interactions with immunosuppressive agents, and anti-cancer medications.

\section{Abbreviation}

TB: Tuberculosis; KTR: Kidney transplant recipient; MTB: Mycobacterium tuberculosis; CT: Computerized tomography; ${ }^{18} \mathrm{~F}$-FDG-PET: ${ }^{18} \mathrm{~F}-$

fluorodeoxyglucose positron-emission tomography; VATS: Video-assisted

thoracoscopic surgery; KT: Kidney transplant; LC: Lung cancer

\section{Supplementary Information}

The online version contains supplementary material available at https://doi. org/10.1186/s12879-021-06350-x.

Additional file 1.

Additional file 2

\section{Acknowledgements}

Not applicable.

\section{Authors' contributions}

GF and NCC conducted the literature review and wrote the draft. LLH and WPX collected clinical data. CMH and WC conceived the study and revised the manuscript. All authors have read and approved the manuscript

\section{Funding}

This work was supported by Key Project of Medical Science and Technology Development Foundation of Nanjing (ZKX18042) and by General Program of Jiangsu Provincial Health Commission Foundation (M2020019). The former was used to support data query and the latter paid the article-processing

\section{Availability of data and materials}

Data relating to this study are contained and presented in this document. Other materials are available from the corresponding authors on reasonable request.

\section{Declarations}

\section{Consent to publication}

Written informed consent was obtained from the patient for publication of this case report and any accompanying images. A copy of the written consent is available for review by the Editor of this journal.

\section{Ethics approval and consent to participate}

This study was approved by the institutional review boards of the Second Hospital of Nanjing (2019-LS-ky005). All authors have read and approved the manuscript.

\section{Competing interests}

The authors declare that they have no competing interests.

\section{Author details}

'Department of Tuberculosis, The Second Hospital of Nanjing, Nanjing University of Chinese Medicine, Nanjing 210003, China. ${ }^{2}$ Department of Respiratory, Xinglong Community Health Center, Nanjing 210019, China ${ }^{3}$ Department of Respiratory and Critical Care Medicine, Jiangsu Province Hospital, the First Affiliated Hospital of Nanjing Medical University, Nanjing, China. ${ }^{4}$ Clinical Research Center, The Second Hospital of Nanjing, Nanjing University of Chinese Medicine, Nanjing 210003, China.
Received: 22 March 2021 Accepted: 24 June 2021

Published online: 01 July 2021

\section{References}

1. Haberal M, Boyvat F, Akdur A, Kırnap M, Özçelik Ü, Yarbuğ Karakayalı F. Surgical complications after kidney transplantation. Exp Clin Transplant. 2016:14(6):587-95.

2. Carvalho JA, Nunes P, Antunes H, Parada B, Tavares da Silva E, Rodrigues L, et al. Surgical complications in kidney transplantation: an overview of a Portuguese reference center. Transplant Proc. 2019;51(5):1590-6. https://doi. org/10.1016/j.transproceed.2019.05.001.

3. Mamishi S, Pourakbari B, Moradzadeh M, van Leeuwen WB, Mahmoudi S. Prevalence of active tuberculosis infection in transplant recipients: a systematic review and meta-analysis. Microb Pathog. 2020;139:103894. https://doi.org/10.1016/j.micpath.2019.103894.

4. Eswarappa M, H.J. GD, John MM, Chennabasappa GK, Siddaiah GM. Tuberculosis in renal transplant recipients: our decade long experience with an opportunistic invader. Indian J Tuberc. 2020;67(1):73-8. https://doi.org/1 0.1016/j.jitb.2019.05.001.

5. Boubaker $\mathrm{K}$, et al. $<\mathrm{i}>$ Mycobacterium tuberculosis</i> Infection following Kidney Transplantation. Biomed Res Int. 2013;2013:347103.

6. Au E, Wong G, Chapman JR. Cancer in kidney transplant recipients. Nat Rev Nephrol. 2018;14(8):508-20. https://doi.org/10.1038/s41581-018-0022-6.

7. Gioco R, Corona D, Agodi A, Privitera F, Barchitta M, Giaquinta A, et al. De novo Cancer incidence and prognosis after kidney transplantation: a single center analysis. Transplant Proc. 2019;51(9):2927-30. https://doi.org/10.1016/ j.transproceed.2019.04.096

8. Muntean A, Lucan M. Immunosuppression in kidney transplantation. Clujul Med. 2013;86(3):177-80.

9. Hang T-X, Fang G, Huang $Y$, Hu CM, Chen W. Misdiagnosis of a multi-organ involvement hematogenous disseminated tuberculosis as metastasis: a case report and literature review. Infect Dis Poverty. 2020;9(1):66. https://doi.org/1 0.1186/s40249-020-00681-8.

10. Liu J, Yan J, Wan Q, Ye Q, Huang Y. The risk factors for tuberculosis in liver or kidney transplant recipients. BMC Infect Dis. 2014;14(1):387. https://doi. org/10.1186/1471-2334-14-387

11. Zhang SX, Liu Y. Primary lung cancer in Chinese renal transplant recipients: a single-center analysis. Nan Fang Yi Ke Da Xue Xue Bao. 2017;37(6):715-20.

12. Rousseau-Gazaniol C, Fraboulet S, Couderc $\amalg$, Kreis H, Borie R, Tricot L, et al. Lung cancer in renal transplant recipients: a case-control study. Lung Cancer. 2017;111:96-100. https://doi.org/10.1016/j.lungcan.2017.07.011.

13. Harding E. WHO global progress report on tuberculosis elimination. Lancet Respir Med. 2020;8(1):19. https://doi.org/10.1016/S2213-2600(19)30418-7.

14. Ho JC, Leung CC. Management of co-existent tuberculosis and lung cancer. Lung Cancer. 2018;122:83-7. https://doi.org/10.1016/j.lungcan.2018.05.030.

15. Molina-Romero C, Arrieta O, Hernández-Pando R. Tuberculosis and lung cancer. Salud Publica Mex. 2019;61(3):286-91. https://doi.org/10.21149/1 0090

16. Keikha M, Esfahani BN. The relationship between tuberculosis and lung Cancer. Adv Biomed Res. 2018:7:58.

17. Brenner DR, Boffetta P, Duell EJ, Bickeboller H, Rosenberger A, McCormack $V$, et al. Previous lung diseases and lung cancer risk: a pooled analysis from the international lung Cancer consortium. Am J Epidemiol. 2012;176(7):57385. https://doi.org/10.1093/aje/kws151.

18. Anastasopoulou A, Ziogas DC, Samarkos M, Kirkwood JM, Gogas H. Reactivation of tuberculosis in cancer patients following administration of immune checkpoint inhibitors: current evidence and clinical practice recommendations. J Immunother Cancer. 2019;7(1):239. https://doi.org/10.11 86/s40425-019-0717-7.

19. Jacobs REA, Gu P, Chachoua A. Reactivation of pulmonary tuberculosis during cancer treatment. Int J Mycobacteriol. 2015;4(4):337-40. https://doi. org/10.1016/j.ijmyco.2015.05.015.

20. Anastasopoulou A, Ziogas DC, Samarkos M, Kirkwood JM, Gogas H. Reactivation of tuberculosis in cancer patients following administration of immune checkpoint inhibitors: current evidence and clinical practice recommendations. J Immunother Cancer. 2019;7(1):239. https://doi.org/10.11 86/s40425-019-0717-7.

21. Anand M, Nayyar E, Concepcion B, Salani M, Schaefer H. Tuberculosis in kidney transplant recipients: a case series. World J Transplant. 2017:7(3):21321. https://doi.org/10.5500/wjt.v7.i3.213. 
22. Ankrah AO, van der Werf TS, de Vries EFJ, Dierckx RAJO, Sathekge MM Glaudemans AWJM. PET/CT imaging of mycobacterium tuberculosis infection. Clin Transl Imaging. 2016;4(2):131-44. https://doi.org/10.1007/s4 0336-016-0164-0.

23. Kiranantawat $\mathrm{N}$, et al. Feasibility and accuracy of CT-guided percutaneous needle biopsy of cavitary pulmonary lesions. Diagn Interv Radiol. 2019;25(6): 435-41. https://doi.org/10.5152/dir.2019.18061.

24. Kiranantawat N, McDermott S, Fintelmann FJ, Montesi SB, Price MC, Digumarthy SR, et al. Clinical role, safety and diagnostic accuracy of percutaneous transthoracic needle biopsy in the evaluation of pulmonary consolidation. Respir Res. 2019;20(1):23. https://doi.org/10.1186/s12931-0190982-5.

25. Higuita LM, Nieto-Ríos JF, Daguer-Gonzalez S, Ocampo-Kohn C, AristizabalAlzate A, Velez-Echeverri C, et al. Tuberculosis in renal transplant patients: the experience of a single center in Medellín-Colombia, 2005-2013. J Bras Nefrol. 2014;36(4):512-8. https://doi.org/10.5935/0101-2800.20140073.

26. Schmidt-Lauber C, Jacobi J, Polifka I, Hilgers KF, Wiesener MS. Suspected colonic cancer turns out to be disseminated tuberculosis in a kidney transplant recipient: a case report. Medicine (Baltimore). 2019;98(36):e16995. https://doi.org/10.1097/MD.0000000000016995.

27. Hirashima T, Tamura Y, Han Y, Hashimoto S, Tanaka A, Shiroyama T, et al. Efficacy and safety of concurrent anti-Cancer and anti-tuberculosis chemotherapy in Cancer patients with active mycobacterium tuberculosis: a retrospective study. BMC Cancer. 2018;18(1):975. https://doi.org/10.1186/s12 885-018-4889-1.

28. Anastasopoulou A, Ziogas DC, Samarkos M, Kirkwood JM, Gogas H. Reactivation of tuberculosis in cancer patients following administration of immune checkpoint inhibitors: current evidence and clinical practice recommendations. J Immunother Cancer. 2019;7(1):239. https://doi.org/10.11 86/s40425-019-0717-7.

\section{Publisher's Note}

Springer Nature remains neutral with regard to jurisdictional claims in published maps and institutional affiliations.

Ready to submit your research? Choose BMC and benefit from:

- fast, convenient online submission

- thorough peer review by experienced researchers in your field

- rapid publication on acceptance

- support for research data, including large and complex data types

- gold Open Access which fosters wider collaboration and increased citations

- maximum visibility for your research: over $100 \mathrm{M}$ website views per year

At $\mathrm{BMC}$, research is always in progress.

Learn more biomedcentral.com/submissions 